\title{
Unsolvability of the knot problem for surface complexes
}

\section{John C. Stillwell}

It is shown that the problem of deciding whether a polygonal curve $c$ in a finite surface complex $K$ is knotted in $K$ is complete recursively enumerable, and hence unsolvable.

We refer to [6] for the definition of a finite surface complex, introductory remarks, and general references. In [6] it was shown that the problem of deciding whether an edge path $c$ in a 2-dimensional simplicial complex $K$ bounds a disc in $K$ is NP-complete. Generalizing to an arbitrary polygonal path $c$ in $K$ gives an equivalent problem, since $K$ may be simplicially subdivided to make $c$ an edge path in polynomial time. Bounding a disc is equivalent to the existence of an isotopy which contracts $c$ to a point without pulling it over any point twice.

In the present paper we discuss the equivalence of simple curves under more general isotopies in $K$, namely simplicial isotopies in an arbitrary simplicial decomposition of $K$. Curves $c_{1}, c_{2}$ are called simplicially isotopic with respect to a simplicial decomposition $\Sigma$ of $K$, if there is a finite sequence of simple edge paths of $\Sigma$,

$$
c_{1}=c^{(1)}, c^{(2)}, \ldots, c^{(k)}=c_{2},
$$

such that $c^{(m+1)}$ is the result of pulling $c^{(m)}$ from one side to the other of a triangle in $\Sigma$. A curve $c$ is called simplicially unknotted with respect to $\Sigma$ if it is simplicially isotopic to a curve which bounds a disc, and unknotted in $K$ if it is simplicially unknotted with respect

Received 13 December 1978. 
to some simplicial decomposition $\Sigma$ of $K$. By the Hauptvermutung for surface complexes, [7], $c$ is unknotted if it is simplicially unknotted in a sufficiently fine simplicial decomposition, for example the $n$th barycentric subdivision for sufficiently large $n$.

The reason we do not use general isotopies in $K$ to define knotting was pointed out by Alexander [1] in the case of classical knots in $R^{3}$. Alexander's example may be adapted to surface complexes using a "book with three leaves" $K$ (see page 133).

The curve $c$ is a trefoil knot when $K$ is embedded in $\mathrm{R}^{3}$; nevertheless the isotopy $(I) \rightarrow(2) \rightarrow(3)$ reduces it to a curve bounding a disc.

It is clear that we can decide whether a curve is unknotted with respect to a given $\Sigma$ by enumerating the finitely many possible simplicial isotopies. (In fact this can be done by a non-deterministic linear bounded Turing machine, or using Savitch's Theorem [4], by a deterministic Turing machine on quadratically bounded tape.) By applying this decision process in successive barycentric subdivisions of $K$ we see that the set of pairs $(K, c)$ for which $c$ is an unknotted polygonal curve in $K$ is recursively enumerable.

We now show that the set is complete recursively enumerable by reducing the word problem for finitely presented groups to it.

THEOREM 1. Given a finite presentation $G$ of a group, and a word $w$ in $G$, we con effectively construct a finite surface complex $K(G)$ and a simple polygonal curve $c(w)$ such that

$4 .:$

$$
c(w) \text { is unknotted in } K(G) \Longleftrightarrow w=1 \text { in } G \text {. }
$$

Proof. $K(G)$ is a slight modification of the complex used by Dehn [2] to realize an arbitrary finitely presented group

$$
G=\left\langle a_{1}, \ldots, a_{n} ; r_{1}, \ldots, r_{m}\right\rangle \text {. }
$$

Dehn takes a bouquet $B$ of circles $a_{1}, \ldots, a_{n}$ to realize the generators, and realizes each relation $r_{j}=1$ by attaching a disc $D_{j}$ along its boundary to the path $r_{j}$ (spelled as a product of $a_{i}$ 's) in $B$. 
(1)

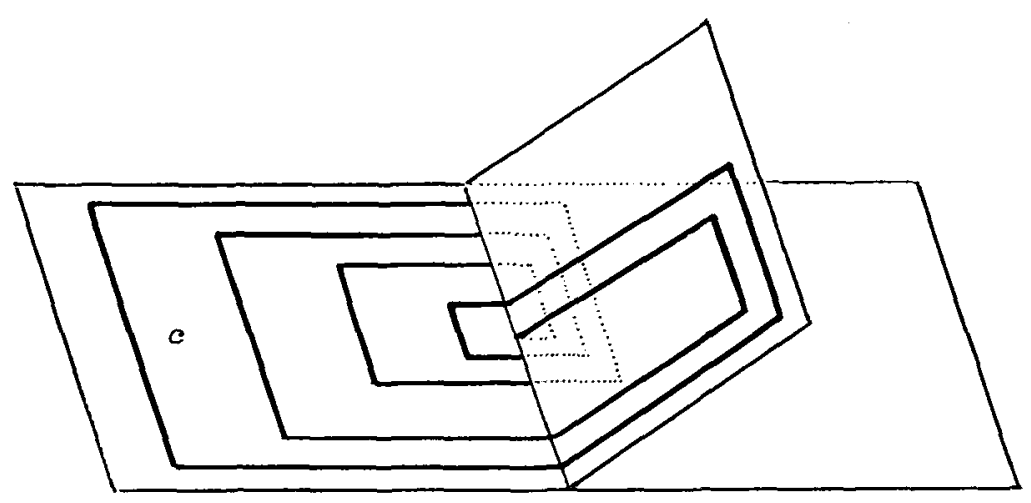

(2)

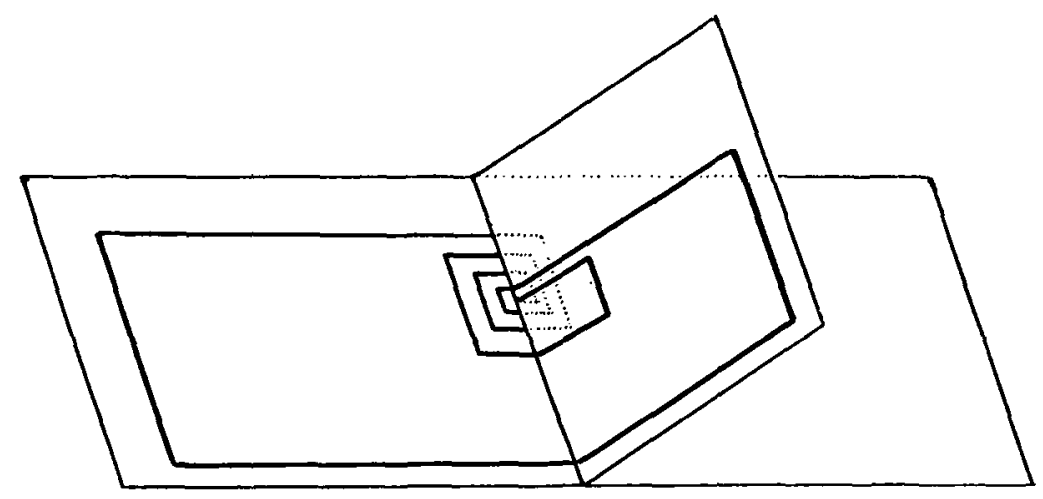

(3)

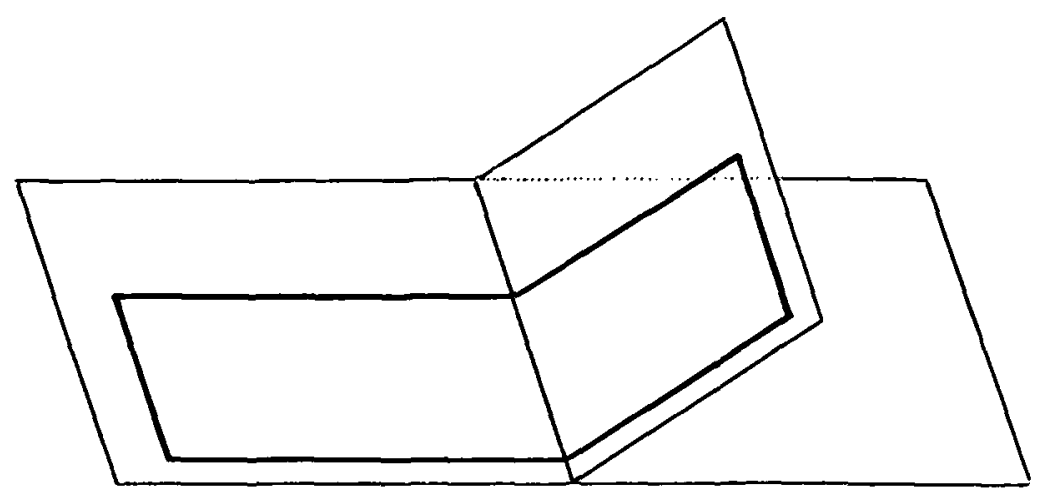


We realize each $a_{i}$ by an annulus $A_{i}$ which has $a_{i}$ as its centre- line, and let the different $A_{i}$ meet along a common transverse segment $[0,1]$ (and nowhere else). Given a word

$$
w=a_{i_{1}}^{\varepsilon_{1}} a_{i_{2}}^{\varepsilon_{2}} \ldots a_{i_{k}}^{\varepsilon_{k}}, \quad \varepsilon_{l}= \pm 1 \text {, }
$$

we construct a simple arc $\alpha(w)$ in $\bigcup_{i} A_{i}$ by taking points $0<P_{1}<P_{2}<\ldots<P_{k+1}<1$ on $[0,1]$ and connecting each $P_{l}$ to $P_{l+1}$ by the "geodesic" (in a natural sense) in $A_{i_{\tau}}$ with orientation implied by $\varepsilon_{l} \cdot$ For example if

$$
w=a_{1} a_{2} a_{2}^{-1} a_{1} a_{2}
$$

then $a(w)$ will resemble the curve in the figure on page 135 . (It is not unique because of the arbitrariness in the choice of $P_{1}, \ldots, P_{k+1}$; however, different $a(w)$ 's will be isotopic - a fact which is exploited below.)

It is clear that any word $w$ is representable by a simple arc in this way, and hence if we attach $[0,1]$ to the top side of a square $S$ which is otherwise disjoint from $U_{i} A_{i}$ we can close $a(w)$ to a simple curve $c(w)$ by running round the other three sides of the square. Furthermore, the fundamental group of $A=\bigcup_{i} A_{i} \cup S$ is the free group generated by $a_{1}, \ldots, a_{n}$, since there is a deformation retraction of $A$ onto the bouquet of circles $U_{i} a_{i}$, and $c(w)$ represents the element $w$.

We now attach a disc $D_{j}$ which will allow us to insert or remove a subarc $a\left(r_{j}\right)$ of a $c(w)$ by an isotopy. Namely, take any points $Q,-R$ with $0<Q<R<1$ and let $\bar{a}\left(r_{j}\right)$ be any fixed $a\left(r_{j}\right)$ which runs from $Q$ to $R$. Then $b\left(r_{j}\right)=\bar{a}\left(r_{j}\right) \cup R Q$ is taken as the boundary of $D_{j}$. Notice that the simple arc $\bar{a}\left(r_{j}\right)$ may be deformed isotopically into the 


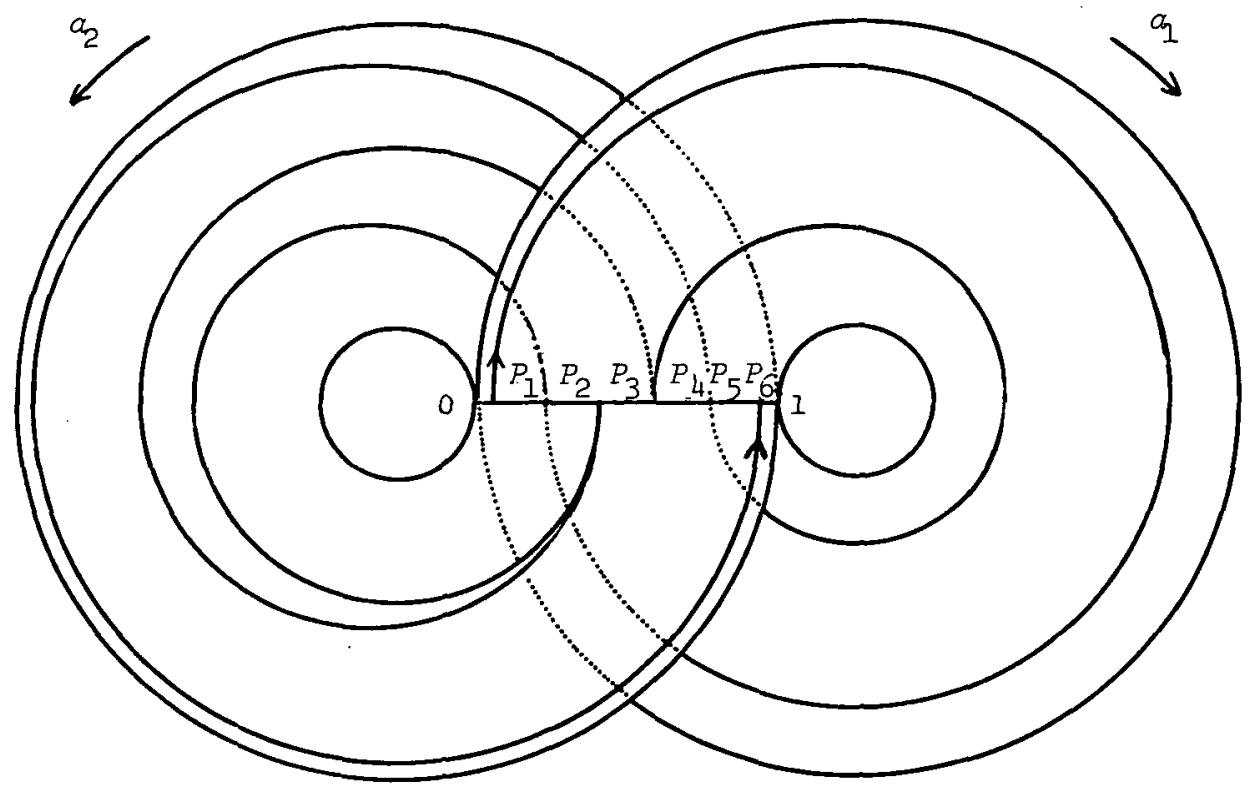

line segment $Q R$ by pulling it across $D_{j}$. We let $K(G)=A \cup \underset{j}{\cup} D_{j}$.

Then to remove a subarc $a\left(r_{j}\right)$ of $c(w)$ we first deform $c(w)$ isotopically so that $a\left(r_{j}\right)$ is carried onto $\bar{a}\left(r_{j}\right)$, then pull $\bar{a}\left(r_{j}\right)$ across $D_{j}$ to the position $Q R$. A further isotopy contracts $Q R$ to a point and gives a curve $c\left(w^{\prime}\right)$ where $w^{\prime}$ is the result of removing $r_{j}$ from $w$. The reverse process simulates the insertion of $r_{j}$ in $w^{\prime}$ to produce $w$. Insertion or removal of trivial relators $a_{i} a_{i}^{-1}$ or $a_{i}^{-1} a_{i}$ can obviously be accomplished by isotopies in $A$ itself.

Since any word $w$ which equals 1 in $G$ can be converted to the 
empty word by a finite sequence of insertions or removals of relators, the corresponding curve $c(w)$ will be convertible to the boundary of the square $S$ by a finite sequence of isotopies of the above type (which can be realized in a sufficiently fine simplicial decomposition of $K(G)$ ) and hence unknotted. On the other hand, it is clear from the Seifert-Van Kampen Theorem [5] that the fundamental group of $K(G)$ is precisely $G$; hence when $w \neq 1$ in $G$ the curve $c(w)$ will not even be homotopic, let alone isotopic, to the boundary of a disc.

COROLLARY. The set of pairs $(K, c)$ for which $c$ is a knotted polygonal curve in a finite 2-dimensional simplicial complex $K$ is not recursively enumerable.

Proof. If it were, the set $\{(K, c) \mid c$ is unknotted in $K\}$ would be recursive, and the construction of Theorem $l$ would yield an algorithm for the word problem for groups.

Another obvious corollary to this theorem is that the problem of deciding whether a polygonal curve in a surface complex is isotopic (in the general sense) to a point is unsolvable. Furthermore, we obtain unsolvability of both problems in a fixed $K(G)$ by choosing a $G$ with unsolvable word problem. This shows that surface complexes constitute an exception to the remark of Haken [3] that isotopy problems are easier to solve than homotopy problems.

\section{References}

[1] J.W. Alexander, "Some problems in topology", Verhandlungen des IntermationaZen Mathematiker-Kongresses, Zürich, 1932. Band I. Bericht und AlZgemeine Vorträge, 249-257 (Orell Füssli, Zürich, Leipzig, ND [1933]; Kraus Reprint Limited, Nendeln, 1967).

[2] M. Dehn, "Über die Topologie des dreidimensionalen Raumes", Math. Ann. $69(1910), 137-168$.

[3] Wolfgang Haken, "Connections between topological and-group theoretical decision problems", Word problems, decision problems and the Burnside problem in group theory, 427-441 (Studies in Logic and the Foundations of Mathematics, - North-Holland, Amsterdam, London, 1973). 
[4] Walter J. Savitch, "Relationship between nondeterministic and deterministic tape complexities", J. Computer System Sci. 4 (1970), 177-192.

[5] H. Seifert und W. Threlfall, Lehrbuch der Topologie (B.G. Teubner, Leipzig, Berlin, 1934. Reprinted Chelsea, New York, 1947).

[6] John C. Stillwell, "Isotopy in surface complexes from the computational viewpoint", Bulz. Austral. Math. Soc. 20 (1979), $1-6$.

[7] E.F. Whittlesey, "Classification of finite 2-complexes", Proc. Amer. Math. Soc. 9 (1958), 841-845.

Department of Mathematics,

Monash University,

Clayton,

victoria. 